\title{
The Influence of Nonrandom Mating on Population Growth
}

\author{
Susanne Schindler, ${ }^{1, \star}$ Peter Neuhaus, ${ }^{2}$ Jean-Michel Gaillard, ${ }^{3}$ and Tim Coulson ${ }^{1}$ \\ 1. University of Oxford, OX1 3PS, United Kingdom; 2. University of Calgary, Calgary, Alberta, T2N 1N4, Canada; 3. Université \\ Claude Bernard Lyon 1, Villeurbanne Cedex, France
}

Submitted October 1, 2012; Accepted March 8, 2013; Electronically published May 22, 2013

\begin{abstract}
When nonrandom mating alters offspring numbers or the distribution of offspring phenotypes, it has the potential to impact the population growth rate. Similarly, sex-specific demographic parameters that influence the availability of mating partners can leave a signature on the population growth rate. We develop a general framework to explore how mating patterns and sex differences influence the population growth rate. We do this by constructing a two-sex integral projection model to explore ways in which altering the mating behavior from random to nonrandom mating (assortative, disassortative, or selection for size) and altering demographic parameters in one or both sexes (growth, survival, and parental contribution to offspring phenotype) affect the population growth rate. We demonstrate our framework using data from a population of Columbian ground squirrels. Our results suggest that the population growth rate is substantially affected when nonrandom mating is linked to sex differences in demographic parameters or parental contributions to offspring phenotype, but interestingly, the effect of the mating pattern alone is rather small. Our results also suggest that the population growth rate of Columbian ground squirrels would increase with the degree of disassortative mating and with the degree of the mating advantage of large individuals.
\end{abstract}

Keywords: maternal effects, paternal effects, sex-specific demographic rates, sex-specific growth rates, negative assortative mating, polygynandry.

\section{Introduction}

Many studies have reported nonrandom mating in sexually reproductive species. Biologists have long been interested in understanding how different mate choices evolve (O’Donald 1960; Parker 1983; Dieckmann and Doebeli 1999; Proulx 2001; Van Doorn et al. 2009; Schindler 2010) and influence population dynamics (Doebeli and Koella 1994; Lindström and Kokko 1998). In this article, we investigate the link between nonrandom mating and the population growth rate. We develop a general framework to demonstrate how assortative mating, disassortative mating, and selection for size could influence the population growth rate. We show that nonrandom mating impacts

* Corresponding author; e-mail: susanne.schindler@zoo.ox.ac.uk.

Am. Nat. 2013. Vol. 182, pp. 28-41. (C) 2013 by The University of Chicago. 0003-0147/2013/18201-54151\$15.00. All rights reserved. DOI: $10.1086 / 670753$ the population growth rate when parental phenotypes determine the distribution of their offsprings' phenotypes. Our results suggest that nonrandom mating, together with sex-specific growth and fertility rates and maternal effects, can leave a substantial signature on population dynamics. We provide a way of exploring the magnitude of these effects.

The causes of nonrandom mating can be diverse. They include individual preferences (e.g., preference for colorbanded mates in zebra finches; Burley et al. 1982; Burley 1986); heterogeneous distributions of a population in space and/or time (e.g., prezygotic isolation in plants via different flowering times); and mechanistic constraints, such as the compatibility of genitalia (e.g. mating of equalsized leaf beetles is more successful than mating of leaf beetles that are different sizes; Brown 1993). Despite the many causes of nonrandom mating, the mating system and mating tactics play a significant role in affecting the relative ability of females and males to mate in relation to their phenotype. The causes and magnitude of nonrandom mating can consequently differ between the sexes, with one sex potentially mating predominantly with a partner of a large size, whereas mating in the other sex is nonselective with respect to size. In cases like this, the magnitude of between-sex differences in nonrandom mating is expected to increase with the intensity of sexual selection, as are differences in the demographic parameters between the sexes. Our approach allows us to investigate how such differences impact the population growth rate, and we consider how a range of nonrandom mating tactics influences the population growth rate when operating in either one or both sexes.

Assortative mating occurs when phenotypically similar individuals are more likely to mate with each other than with individuals of dissimilar phenotypes. Examples include the strawberry poison frog, in which mating occurs mostly between individuals of the same color morph (Summers et al. 1999). Similarly, Darwin's finches mate assortatively with respect to beak size (Huber et al. 2007), and leaf beetles mate assortatively with respect to body size (Brown 1993). In several species (e.g., among humans, but especially among monogamous species, such as fla- 
mingos and albatrosses), the character with the highest assortment coefficient is age (Price and Vandenberg 1980). The converse of assortative mating is disassortative mating, in which a negative correlation between the traits of a mating pair is observed. Examples include a preference for partners that have dissimilar genotypes at the major histocompatibility complex, a gene region influencing the immune system (Wedekind et al. 1995). Disassortative mating based on dissimilar plumage has also been reported in the white-throated sparrow (Houtman and Falls 1994).

A third prominent example of nonrandom mating is when the individual mating probability increases with the value of a trait, such as size. This is observed in haremkeeping males, such as gorilla (Kappeler and Schaik 2004) and red deer (Clutton-Brock et al. 1979; Pemberton et al. 1992), and in males with lek-based mating behavior (Höglund and Lundberg 1987).

Nonrandom mating can both increase and decrease the fitness of an individual and is consequently likely to influence the population as a whole. There are various mechanisms via which the population can be impacted. Nonrandom mating might increase female fertility and the number of offspring produced; females choosing males that provide direct benefits, including resource provisioning or parental care, can increase their reproductive success (Bateson 1983; Andersson 1994). Alternatively, when dimorphism is very pronounced within a mating pair, several costs can occur, including injuries when mating. Another mechanism that can link nonrandom mating and population dynamics is the distribution of offspring characters, which in turn influence offspring survival and, consequently, population growth. This "good genes" theory, which proposes that mates influence the quality of their offspring, has been studied both theoretically (Kirkpatrick 1996; Mays and Hill 2004) and empirically (Andersson 1994; Møller and Alatalo 1999; Byers and Waits 2006; Garcia-Gonzalez and Simmons 2011). To our knowledge, there is no analysis that has studied population dynamic effects via inheritance and growth.

There is substantial empirical evidence that variation in mating systems and mating tactics occurs among species, among populations within species, and among individuals within populations (Lott 1991). In this article, we focus on how population growth is influenced by differences in mating behavior among populations within a species. However, our framework could be extended to explore differences between individuals and between species. Our approach is to develop a deterministic two-sex integral projection model (IPM) that includes a function describing the mating pattern. We specify the mating function such that we can alter it to model assortative mating, disassortative mating, and selection for size. From the model, we calculate the asymptotic growth rate $\lambda=$ $\lim _{t \rightarrow \infty} N_{t+1} / N_{t}$, the limit of the ratio of population sizes at consecutive time steps. This is the appropriate measure of mean fitness for a deterministic environment (Fisher 1930). We investigate how $\lambda$ changes when we alter the mating behavior as well as other functions in the model.

There is plenty of support for alternative mating tactics within the same species (Lott 1991), variation in mating behavior within a population (Byers et al. 1994; Byers and Waits 2006), and variation in social systems within a species (Lott 1984), but we are not aware of any system that displays a range of mating patterns from assortative through random to disassortative. However, because mating behavior is not species specific, we can still test the effects of varying mating patterns by exploring a range of mating patterns in the same species, even though they may not all be observed in a single population. We demonstrate how our approach can be used by parameterizing a model with data from a Columbian ground squirrel population (Urocitellus columbianus). Squirrels are quite plastic in their mating behavior, which has potential for mate choice in both sexes. The Columbian ground squirrels exhibit a complex polygynandrous mating system and mating tactics that are based on territoriality (Balmer 2010). Individuals of both sexes can mate with multiple partners, and a high proportion of litters have multiple paternities (Raveh et al. 2010). Also, males and females of the Columbian ground squirrel exhibit similar demographic characteristics. We start by assuming identical demographic characteristics, but we relax this assumption later on.

\section{The Model}

IPMs are discrete time models of the dynamics of continuous traits, like body size or weight (Easterling et al. 2000). The number of individuals with a certain trait can be projected in time:

$$
n(y, t+1)=\int[p(x, y)+q(x, y)] n(x, t) \mathrm{d} x .
$$

Here, the number (or relative frequency) of trait $y$ at time $t+1$ is denoted by $n(y, t+1)$. It is iterated from trait numbers (or relative frequencies) at time $t$ if we know the probability that an individual of trait $x$ survives and develops to trait $y$ within a single time step (denoted by $p(x, y))$ and if we know the contribution of an individual with trait $x$ to offspring with trait $y$ (denoted by $q(x, y)$ ).

We introduce a two-sex model and split $n(x, t)$ into its contributions from the female sex, $n_{\mathrm{f}}(x, t)$, and from the male sex, $n_{\mathrm{m}}(x, t), n(x, t)=n_{\mathrm{f}}(x, t)+n_{\mathrm{m}}(x, t)$.

Mating behavior is sufficiently described by the set of mating probabilities for each parent combination. We de- 
note the term to which the actual mating probability between $x$-trait and $y$-trait individuals is proportional by $m(x, y)$ and require that $\int_{y_{\min }}^{\infty} m(x, y) \mathrm{d} y=1$ for all $x$. If abundances are not an issue, this ensures that every adult female mates with at least one male. This setup is suitable for a polygynandrous mating system in which both females and males can mate with multiple partners. In contrast to females, we do not require that every male is mated. This allows more variability in the mating success of males than in females, and it also allows that one male can inseminate all females.

In addition to specifying mating probabilities, we need to specify the number of offspring per breeding event, denoted by $R(x, y)$, and the distribution of offspring characters at birth. The offspring distribution is denoted by $f(x \mid y, z)$, which gives the probability that a newborn has trait $x$ if its parents have traits $y$ and $z$. Because $f(x \mid y, z)$ is a conditional probability, it holds that $\int f(x \mid y, z) \mathrm{d} x=$ 1 . The sex ratio is denoted by $s$, which gives the fraction of female offspring, whereas $(1-s)$ gives the fraction of male offspring. With these notations, the number of $x$ trait females and males can be calculated by

$$
\begin{gathered}
n_{\mathrm{f}}(x, t+1)=\int p(x, y) n_{\mathrm{f}}(y, t) \mathrm{d} y+\ldots \\
+s C_{n_{\mathrm{f}} n_{\mathrm{m}}} \int f(x \mid y, z) m(y, z) n_{f}(y, t) n_{m}(z, t) R(y, z) \mathrm{d} y \mathrm{~d} z, \\
n_{\mathrm{m}}(x, t+1)=\int p(x, y) n_{\mathrm{m}}(y, t) \mathrm{d} y+\ldots \\
+(1-s) C_{n_{\mathrm{f}}, n_{\mathrm{m}}} \int f(x \mid y, z) m(y, z) n_{\mathrm{f}}(y, t) n_{\mathrm{m}}(z, t) R(y, z) \mathrm{d} y \mathrm{~d} z .
\end{gathered}
$$

Both equations consist of two summations. The first captures the fate of individuals alive at time $t$ : whether they survive to time $t+1$ and, if they do, their trait value at that time. The second sum captures newborns. The number of newborns is calculated by multiplying the mating function, $m(y, z)$, with the number of $y$-trait females, the number of $z$-trait males, and the litter size of the mating pair, $R(y, z)$, before multiplying that with the probability that their offspring have trait $x$ at birth, $f(x \mid y, z)$, and summing over all possible parent combinations (i.e., over $y$ and $z$ ). In all functions that use both maternal and paternal phenotypes, the first phenotype belongs to the mother, and the second belongs to the father. For example, $R(y, z)$ is the number of offspring of female $y$ with male $z$. The same holds for functions $m(y, z)$ and $f(x \mid y, z)$.

The normalization constant $C_{n_{\mathrm{f}}, n_{\mathrm{m}}}$ in equations (2a) and (2b) is set to

$$
C_{n_{\mathrm{f}}, n_{\mathrm{m}}}=\frac{\int_{y_{\min }}^{\infty} n_{\mathrm{f}}(y, t) \mathrm{d} y}{\int_{0}^{\infty} m(y, z) n_{\mathrm{f}}(y, t) n_{\mathrm{m}}(z, t) \mathrm{d} y \mathrm{~d} z},
$$

with $y_{\min }$ denoting the minimal trait value for reproduc- tion. For example, $y_{\min }$ could be a minimum body weight at which females reproduce. Equation (3) imposes a constraint, such that the overall number of birth events or parturitions is set to the overall number of females that are large enough to reproduce. Depending on the mating behavior of the species under study, $C_{n_{\mathrm{f}}, n_{\mathrm{m}}}$ can be modified in various ways: it is, for instance, straightforward to alter it such that the number of birth events is determined by the number of the rarer sex, as in monogamous species (see also birth or marriage functions in human demography; McFarland 1972; Caswell 2001). Equation (3) also defines scaling properties of the model. With our choice of $C_{n_{5} n_{\mathrm{m}}}$, the number of birth events and offspring scales with the number of females but not with the number of males. That means that doubling the female population causes a doubling of offspring. Scaling the male population by a factor, in contrast, has no effect on birth events and offspring numbers, except when there are no males at all. In that case, there are no offspring either, and the population will become extinct when the last female dies.

\section{Modeling Mating}

Our aim is to study the sensitivity of the population growth rate $\lambda$ to nonrandom mating. We focus on three types of nonrandom mating: assortative mating, disassortative mating, and selection for size. In a population whose members mate disassortatively with respect to a trait such as weight, there is a negative correlation between the weights of partners. That means that small individuals prefer large partners. In assortatively mating populations, the correlation coefficient is positive, meaning that individuals prefer partners of similar weight. When there is selection for size, the mating function $m(y, z)$ increases either with $y$ or with $y$ and $z$ together, depending on whether there is sexual selection in one or in both sexes. We focus on sexual selection in males only, because this is often reported for mammals and other vertebrates (Andersson 1994).

We model the three types of nonrandom mating with three mating functions $m_{\rho}^{\mathrm{d}}$ ("d" for disassortative), $m_{\rho}^{\mathrm{a}}$ ("a" for assortative), and $m_{\rho}^{s}$ ("s" for sexual selection). The parameter $\rho$ determines the strength of nonrandom mating. The mating functions are given with

$$
\begin{aligned}
& m_{\rho}^{\mathrm{d}}(y, z)= \begin{cases}1 / 2 \mathrm{e}^{\left[-\left(y_{\max }-y_{\min }\right)^{2}-(y-z)^{2} / 10,000\right] \rho} & \text { if } y, z \geq y_{\min } \\
0 & \text { otherwise }\end{cases} \\
& m_{\rho}^{\mathrm{a}}(y, z)= \begin{cases}1 / 2 \mathrm{e}^{\left[-(y-z)^{2} / 5,000\right] \rho} & \text { if } y, z \geq y_{\min } \\
0 & \text { otherwise, }\end{cases} \\
& m_{\rho}^{\mathrm{s}}(y, z)= \begin{cases}1 / 2 \mathrm{e}^{[y / 50] \rho} & \text { if } y, z \geq y_{\min } \\
0 & \text { otherwise. }\end{cases}
\end{aligned}
$$

The parameter $y_{\max }$ in equation (4) corresponds to the maximum possible trait value, and the parameter $y_{\min }$ in 
equations (4)-(6) corresponds to the minimal trait value required for reproduction. We chose the exponential function in equations (4)-(6) because its smoothness and positivity are suitable for the use as a probability, and it allows differentiation. The mating function $m_{\rho}^{\mathrm{d}}$ has two maxima when $y$ and $z$ are maximally different. This is disassortative mating. The function $m_{\rho}^{\mathrm{a}}$ has its maxima along the line $y=z$ (i.e., when partners have the same trait values). This is assortative mating. The function $m_{\rho}^{\text {s }}$ has its maxima along the line $y=y_{\max }$. This is selection for large males.

If the parameter $\rho$ is set to 0 , then $m_{0}^{\mathrm{d}}, m_{0}^{\mathrm{a}}$, and $m_{0}^{\mathrm{s}}$ correspond to random mating, and it holds $m_{0}^{\mathrm{d}}=$ $m_{0}^{\mathrm{a}}=m_{0}^{\mathrm{s}}=m_{0}$. A biologically meaningful range of $\rho$ is from 0 to 0.1 , because the shapes of $m_{\rho}^{\mathrm{d}}, m_{\rho}^{\mathrm{a}}$, and $m_{\rho}^{\mathrm{s}}$ then adequately capture nonrandom mating (see, e.g., fig. 1 for $\rho=0.05$ ). For other values of $\rho$, the peaks of the mating functions will be too steep to represent meaningful mating behavior. It is not clear how an empirically estimated correlation coefficient between traits of mating partners could translate into $\rho$; in our model, the value $\rho=0.1$ corresponds to strongly disassortative mating in $m_{\rho}^{\mathrm{d}}$, to strongly assortative mating in $m_{\rho}^{\mathrm{a}}$, and to strong sexual selection in $m_{\rho}^{s}$. Depending on the nonrandom mating under investigation, one of the functions $m_{\rho}^{\mathrm{d}}, m_{\rho}^{\mathrm{a}}$, or $m_{\rho}^{\mathrm{s}}$ replaces $m$ in equations (2) and (3).

The factors 10,000 in equation (4), 5,000 in equation (5), and 50 in equation (6) have been chosen such that, for a given $\rho$, the distances of $m_{\rho}^{\mathrm{d}}, m_{\rho}^{\mathrm{a}}$, and $m_{\rho}^{\mathrm{s}}$ to $m_{0}$ are approximately the same. A measure of that distance is the sum of absolute differences:

$$
\begin{aligned}
& \int\left|m_{\rho}^{\mathrm{d}}(x, y)-m_{0}(x, y)\right| \mathrm{d} x \mathrm{~d} y \\
& \approx \int\left|m_{\rho}^{\mathrm{a}}(x, y)-m_{0}(x, y)\right| \mathrm{d} x \mathrm{~d} y \\
& \approx \int\left|m_{\rho}^{\mathrm{s}}(x, y)-m_{0}(x, y)\right| \mathrm{d} x \mathrm{~d} y .
\end{aligned}
$$

By ensuring that these differences are approximately the same, we make certain that the difference of change in $\lambda$ is not primarily caused by different magnitudes of change in $m_{\rho}^{\mathrm{d}}, m_{\rho}^{\mathrm{a}}$, and $m_{\rho}^{\mathrm{s}}$ to $m_{0}$.

\section{Sex Differences}

To quantify the effect of nonrandom mating on the population growth rate, we have assumed so far that there are no sex differences in the species' demographic characteristics. That means we set both parents to have the same demographic parameters, to have the same growth or developmental rates, and to contribute equally to the phenotype of their offspring. However, the effect of nonran- dom mating depends on sex differences, and so, in a next step, we systematically alter these assumptions.

A summary of all parameters and functions used can be found in tables B1 and B2 in appendix B.

Lower Male Survival Rates. Males experience higher mortality rates than females in several mammalian species (Toïgo and Gaillard 2003; Clutton-Brock and Isvaran 2007), usually when the mating system is polygynous or under harsh environmental conditions. In our model, we lower the survival probability of males in the survival function $p_{s}$. Reduced male survival rates are tuned by a parameter $\varepsilon$ that ranges from 0 (no reduced male survival rates) to 0.5 (slightly reduced male survival rates). The survival function $p_{\mathrm{s}}$ for males is replaced by $p_{\mathrm{s}}^{\prime}$ :

$$
p_{\mathrm{s}}^{\prime}(x)=\frac{\mathrm{e}^{a+b x-\varepsilon}}{1+\mathrm{e}^{a+b x-\varepsilon}}
$$

with $a$ and $b$ fitted to survival data of the species of interest. Combined with $p_{\mathrm{g}}$, the growth function, we have now a female kernel $p_{\mathrm{f}}(x, y)=p_{\mathrm{s}}(x) p_{\mathrm{g}}(y \mid x)$ and a male kernel $p_{\mathrm{m}}(x, y)=p_{\mathrm{s}}^{\prime}(x) p_{\mathrm{g}}(y \mid x)$. The kernel $p_{\mathrm{f}}(x, y)$ replaces $p(x, y)$ in equation $(2 \mathrm{a})$ and $p_{\mathrm{m}}(x, y)$ replaces $p(x, y)$ in equation $(2 \mathrm{~b})$.

We lower male survival rates without altering male growth rates, an aspect that differs from most natural populations where lower male survival rates usually correlate with higher male growth rates (Toïgo and Gaillard 2003) and where males grow for longer than females when the intensity of sexual selection increases (Jarman 1983). However, we alter both rates independently, because we want to identify single causes that change the influence of nonrandom mating. A compound effect can be studied in our framework by jointly applying this and the following model variant (i.e., replacing $p_{\mathrm{s}}$ and $\tilde{p}_{\mathrm{g}}$ by $p_{\mathrm{s}}^{\prime}$ and $\tilde{p}_{\mathrm{g}}^{\prime}$ at the same time; see below).

Higher Male Growth Rates. Males of species with sexual size dimorphism often attain their greater body size relative to females through higher growth rates. In our model, we increase male growth rates by introducing parameter $g$, which ranges from $g=0$ (no increased growth relative to females) to $g=10$ (strongly increased male growth rates). This parameter enters the prenormalized Gaussian growth function $\tilde{p}^{\prime}(x, y) g$ via $\mu^{\prime}(x)=c+d x+g$. Again, we have a female kernel $p_{\mathrm{f}}(x, y)=p_{\mathrm{s}}(x) p_{\mathrm{g}}(y \mid x)$ (replacing $p(x, y)$ in eq. [2a]) and a male kernel $p_{\mathrm{m}}(x, y)=p_{\mathrm{s}}(x) p^{\prime}(y \mid x)$ (replacing $p(x, y)$ in eq. [2b]).

Maternal Body Condition Influences Litter Size. The number of offspring a female produces can be condition dependent (Morris 1998; Descamps et al. 2011) and often 
depends on maternal body weight (Sadleir 1969). We introduce a small amount of size dependence to the number of offspring a female produces with parameter $v$ ranging from $v=0$ (no dependence on maternal condition) to $v=0.005$ (the number of offspring increases with maternal weight):
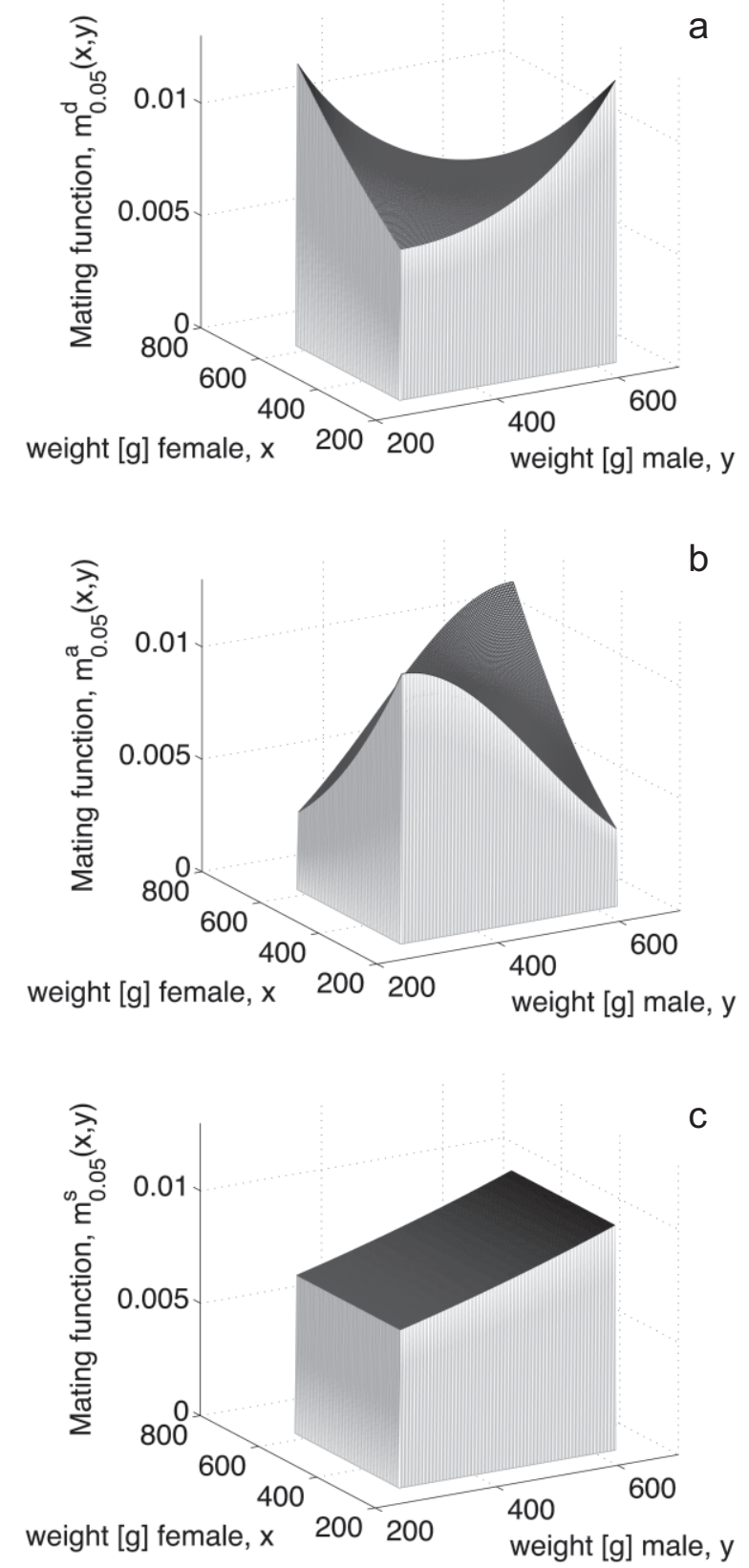

Figure 1: Mating functions $m_{\rho}^{\mathrm{d}}, m_{\rho}^{\mathrm{a}}$ and $m_{\rho}^{\mathrm{s}}$ given in equations (4)(6) for $\rho=0.05$. $a$, Disassortative mating; $b$, assortative mating; $c$, selection for size in one parent. The normalization $\int m(x, y) \mathrm{d} y=1$ for all $x$ causes the asymmetries in the function plotted in $a$ and $b$. See "The Model" for justification of this normalization.

$$
R^{\prime}(x, y)= \begin{cases}r\left[1+v\left(x-y_{\min }\right)\right] & \text { if } x, y \geq y_{\min } \\ 0 & \text { otherwise. }\end{cases}
$$

The parameters $r$ and $y_{\min }$ fit the data and the offspring function $R^{\prime}(x, y)$ replaces $R(x, y)$ in equations (2).

Maternal and Paternal Effects. As the fourth and last sex difference, we introduce maternal effects on offspring size. Even though environmentally driven maternal effects play an important role, especially in mammals with strong mother-offspring bonds during the energy-consuming lactation period, we focus on maternal genetic effects as defined in Cheverud and Wolf (2009), because our framework is deterministic. Parameter $w$ models the degree by which one parent influences offspring phenotype more than the other. The probability that an offspring weighs $x$ is Gaussian distributed, with the mean determined by the weighted average $w y+(1-w) z$. The mean $\mu(y, z)$ of the inheritance function $f(x \mid y, z)$ is replaced by $\mu^{\prime}(y, z)=$ $e+h[w y+(1-w) z]$, and parameters $e$ and $h$ are fitted to the data. The parameter $w$ ranges from $w=0$ (paternal influence only) to $w=1$ (maternal influence only). The new inheritance function $f^{\prime}$ models maternal effects when $w$ departs from 0.5 and replaces $f$ in equations (2). If $w=0.5$, then $f(x \mid y, z)=f^{\prime}(x \mid y, z)$.

An Example: The Columbian Ground Squirrel. Having outlined our framework, we now showcase it using an IPM parametrized for a population of Columbian ground squirrels Urocitellus columbianus. We focus on this rodent because, first, their polygynandrous mating system is complex, allowing intraspecific variation in mating tactics and mate choice in both sexes. Second, females and males of this species have similar demographic characteristics. Therefore, we can realistically assume in our model that there are no sex differences before relaxing this assumption in various ways. The measurements of the Columbian ground squirrel have been taken from 1994 to 1998 from the Sheep River Provincial Park in the foothills of the Rocky Mountains (elevation, 1,500 m), in southwestern Alberta, Canada. We collected data on female spring emergence weight, female survival to the next year, the weight of female survivors the following spring, litter size at weaning (age, 27 days), and offspring weights at weaning for male and female offspring. All weights are taken within 1-2 days of the event (spring emergence or natal emergence) using a Pesola spring scale rounded to the nearest $5 \mathrm{~g}$. Input functions for a one-sex IPM for the Columbian ground squirrel have been parametrized by $\mathrm{T}$. Coulson and P. Neuhaus (unpublished data). We base our model on these functions (see app. A). 


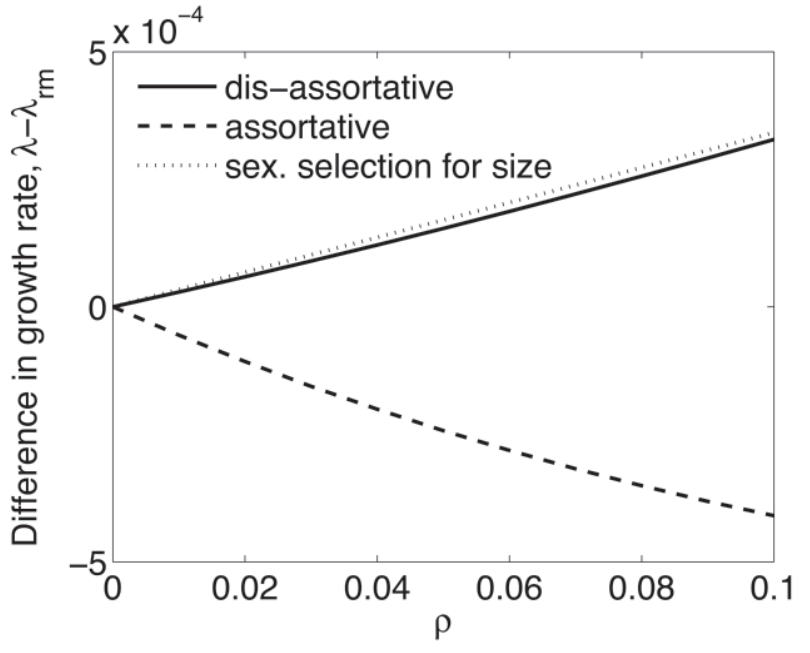

Figure 2: Under random mating (i.e., $\rho=0$ ), the population grows at the rate $\lambda_{\mathrm{rm}}=1.05$. Plot shows the difference in growth rate, $\lambda-\lambda_{\mathrm{rm}}$, when $\rho$ is varied from 0 to 0.1 . Disassortative mating and selection for size increase the growth rate of a population, whereas assortative mating decreases it.

\section{Results for the Columbian Ground Squirrel}

The two-sex IPM given in equations (2) with random mating and inheritance function $f_{2}$ (see "Inheritance" in app. A) gives a population growth rate of $\lambda_{\mathrm{rm}}=1.05$. Values for $\lambda$ are calculated after iterating equations (2) until the population is stable, meaning that its composition remains constant.

\section{Nonrandom Mating and Population Growth}

Selection for size and disassortative mating increase $\lambda$, whereas assortative mating decreases it. Although the independent effects of changing the mating pattern are rather small, we can show that, if the mating pattern and demographic parameters are linked, then the effects can be substantial. Figure 2 plots the absolute difference of $\lambda$ attained under the three types of nonrandom mating compared with random mating. Let $\lambda_{\mathrm{rm}}$ denote the growth rate under random mating, so figure 2 plots $\lambda-\lambda_{\mathrm{rm}}$. The mean population weight changes accordingly with $\lambda$. That means that population mean body weight increases with the strength of disassortative mating and selection for size, whereas mean body weight decreases with the strength of assortative mating.

In other words, increasing the nonrandomness of mating changes $\lambda$ by a fixed percentage. In the case of disassortative mating, an increase of $\rho$ by 0.01 leads to a $0.0014 \%$ increase of $\lambda$; in the case of assortative mating, the decrease of $\lambda$ is $0.0012 \%$; and in the case of selection for size, the increase is $0.0016 \%$.

\section{Changes in Single Mating Probabilities}

If we assume random mating, what would be the effect of an increase in the mating probability of a single mating pair? Figure 3 plots $\lambda-\lambda_{\mathrm{rm}}$ scaled by the distance from random mating (i.e., the relative change in $\lambda$ ):

$$
\frac{\lambda-\lambda_{\mathrm{rm}}}{\int\left|m^{\prime}(x, y)-m_{0}(x, y)\right| \mathrm{d} x \mathrm{~d} y},
$$

when going from random mating to a situation where one parental phenotype combination is slightly more likely than others. Thus figure 3 plots the sensitivity of $\lambda$ to the mating probabilities. Figure 3 shows that, when the mating probability of small parents increases, the population growth decreases, whereas when the mating probability of bigger parents increases, then $\lambda$ also increases. This is because bigger parents produce bigger offspring, which then survive at a higher rate. In other words, changing the mating pattern alters offspring survival, which impacts $\lambda$.

Another signature of changes in single mating probabilities is found in figure 3 in the region for very heavy males (i.e., male weight of 500-700 g). The population distribution for both sexes approaches zero, so the nonzero sensitivity of $\lambda$ in that region is surprising. It is due to the normalization $\int m(x, y) \mathrm{d} y=1$, which means that increasing the mating probability of female $x$ and male $y$ decreases

$$
\rho=0.05
$$

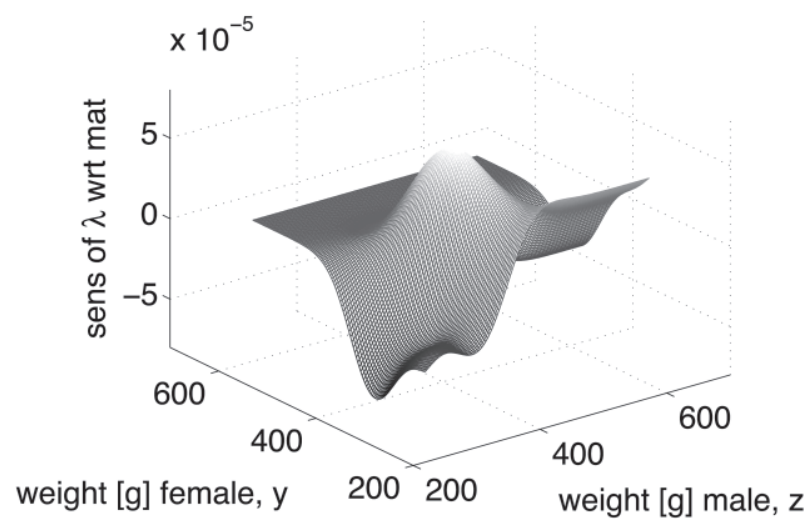

Figure 3: Sensitivity of the growth rate to changes in the mating function for random mating. Plot shows approximation of $\partial \lambda / \partial m_{0}(y, z)$ by $\lambda-\lambda_{\mathrm{rm}} / \int\left|m^{\prime}(y, z)-m_{0}(y, z)\right| \mathrm{d} y \mathrm{~d} z$, where $\lambda_{\mathrm{rm}}=$ 1.05 is the growth rate under random mating, and $\lambda$ is the growth rate when the matrix entry $m_{0}(y, z)$ is increased by $1 \%$ and other entries are reduced to fulfill normalization $\int m^{\prime}(y, z) \mathrm{d} z=1$. The perturbed mating function is named $m^{\prime}$. 
the mating probabilities of that female with all other males. It also means that these probabilities are smaller than for any other mate combination with the exception of $(x, y)$. For males below $500 \mathrm{~g}$, the abundances overlay this effect, but this fails when there are no males of that weight class left. Therefore, we have a positive sensitivity for males over $500 \mathrm{~g}$ and lightweight females (because mating probabilities of lightweight females are reduced in favor of heavier females), and we have a negative sensitivity for males over $500 \mathrm{~g}$ and heavy females (because mating probabilities of heavy females are reduced in favor of more lightweight females).

\section{Nonrandom Mating and Sex Differences}

The type of nonrandom mating has a greater influence on $\lambda$ when we relax the assumption of identical fertility and survival patterns in both sexes. In our initial model, both parents had the same demographic, growth, and inheritance rates. Breaking these symmetries, we find that nonrandom mating and sex differences can have a large impact on the population growth rate (figs. 4, 5).

Lower Male Survival Rates. Lowering male survival rates has a negative impact on population growth. This is surprising, because two-sex matrix models show the opposite result (if structured as in Usher 1972). In addition, the number of birth events is not influenced by the number of males. The smaller the male survival rate is (i.e., the bigger parameter $\varepsilon$ ), the smaller $\lambda$ (fig. $4 a$ ). In addition, the impact of nonrandom mating is amplified by parameter $\varepsilon$ (fig. $4 b$ ). That means that the more male and female survival rates differ (i.e., the more asymmetric the system is regarding survival), the more the growth rate either decreases (assortative mating) or increases (disassortative mating and selection for size) as the mating pattern is altered.

Higher Male Growth Rates. Increasing male growth rates increases population growth rates (fig. 4c). As before, selection for size and disassortative mating increases population growth, and assortative mating decreases it (fig. $4 c$ ). The parameter $g$ enhances the effect of selection for size and dampens to a smaller extent the effect of disassortative and assortative mating (fig. $4 d$ ). So, the more male and female growth rates differ (i.e., the more the system is asymmetric), the more selection for size increases $\lambda$.

Sex-Specific Fertility Rates. Parameter $v$ gives the slope with which the number of offspring depends on the mothers' body weight. The population growth rate increases with $v$, because offspring numbers increase with $v$ (fig. $5 a$ ).
Again, in the case of selection for size and for disassortative mating, increasing $v$ increases the population growth rate (fig. 5a), whereas assortative mating again decreases it. The bigger $v$ is, the stronger the impact of nonrandom mating for disassortative mating and assortative mating is, whereas the magnitude of the effect is constant in $v$ for selection for size (fig. $5 b$ ).

Maternal and Paternal Effects. We model maternal effects with parameter $w$ in $f_{2}^{\prime}$. The stronger the maternal effect (i.e., the bigger $|w-0.5|$ ), the bigger the effect of nonrandom mating on $\lambda$ (fig. $5 c$ ). For assortative mating and selection for size, the growth rate $\lambda$ rises with the paternal influence on juvenile emergence weight, reaching the maximum when fathers alone pass on their phenotype (fig. $5 c$ ). In contrast, for disassortative mating, $\lambda$ decreases with paternal influence.

\section{Discussion}

To understand the evolution of nonrandom mating, researchers have theoretically investigated the conditions under which a specific mating pattern can invade a population with an alternative strategy (Dieckmann and Doebeli 1999; Proulx 2001; Proulx and Servedio 2009). Empirical work has addressed the benefits and causes of observed nonrandom mating patterns (Brown 1993; Thompson et al. 1997). Less is known about how nonrandom mating relates to the growth rate of a population (but see Doebeli and Koella 1994; Lindström and Kokko 1998). To address the link between nonrandom mating and a summary statistic of population dynamics, we developed a framework that easily allowed us to change mating probabilities and to calculate the population growth rate. Our results show that selection for size and disassortative mating generally increase the growth rate, whereas assortative mating decreases it. The effect of the mating pattern alone is small (much less than $1 \%$ change in $\lambda$ ), which in itself is a novel and surprising result. In addition, the effect can be important for long-lived species with low $r_{\text {max }}$, and the effect can be substantial (up to $2 \%$ ) when a change in mating pattern is linked to a change in demographic parameters. In deterministic, density-independent populations, sexual selection for size and disassortative mating would consequently typically invade populations of individuals exhibiting assortative mating preferences. This is somewhat paradoxical, because empirical studies more often report assortative mating in respect to size, rather than disassortative mating. However, in natural populations, assortative mating is primarily caused by availability (spatial or temporal) and mechanistic constraints (Crespi 1989; Brown 1993; Jennions and Petrie 1997). These processes do not enter our model. 

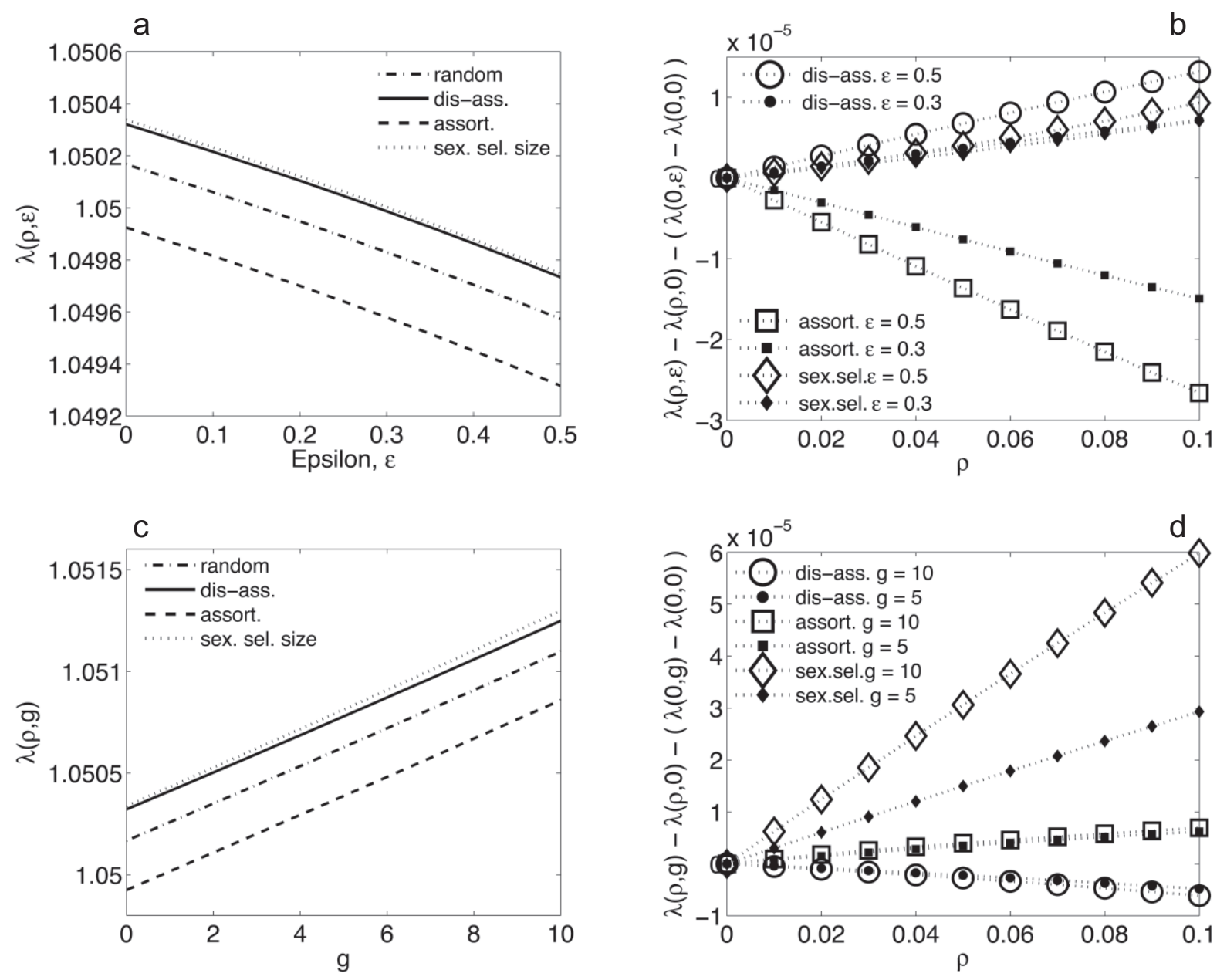

Figure 4: $a$, Population growth $\lambda(\rho, \varepsilon)$ for $\rho=0.05$ and $\varepsilon$ varying along the abscissa. Growth rate $\lambda(0.05, \varepsilon)$ decreases with $\varepsilon$ for all nonrandom mating scenarios. $b$, Difference of growth rate $\lambda(\rho, \varepsilon)$ when $\varepsilon$ is raised from $\varepsilon=0$ to $\varepsilon=0.3$ (small markers) and when $\varepsilon$ is raised from $\varepsilon=0$ to $\varepsilon=0.5$ (large markers) for three nonrandom mating scenarios (disassortative $=$ circle marker; assortative $=$ square; selection for size $=$ diamond). Strength, $\rho$, of nonrandom mating varies along the abscissa. The bigger $\varepsilon$, the stronger the magnitude of the effect of nonrandom mating, but the direction of the effect is the same as in the standard model (fig. 2) in which males have the same survival rates as females. $c$, Population growth rate $\lambda(0.05, g)$ for $\rho=0.05$ increases with $g$ for random mating and nonrandom mating alike. $d$, Difference in growth rate $\lambda(\rho, g)$ when $g$ is raised from $g=0$ to $g=5$ (small markers) and when $g$ is raised from $g=0$ to $g=10$ for three types of mating scenarios (coding as in $b$ ). With selection for size, the magnitude of the effect is increased by asymmetric growth rates, whereas with disassortative and assortative mating the effect is reversed. That means that for disassortative mating, however, it increases $\lambda$, the magnitude of the increase reduces with $g$.

Any change in the growth rate of our model is directly due to nonrandom mating, because other parameters are kept constant while the mating function is altered. For example, as we set the number of births equal to the number of reproducing females, changing the mating behavior from random to nonrandom only changes the distribution of mating pairs. In this scenario, the number of offspring a female produces is constant across all mating pairs, and the way the mating system impacts the population growth rate is through the distribution of offspring sizes. For example, an increase in mean juvenile size increases juvenile survival and consequently increases the population growth rate.

The reason why disassortative mating generally increases mean juvenile emergence weight, whereas assortative mating decreases it, lies in the weight distribution of mating partners. The mating pair distribution is proportional to $m(x, y) n_{\mathrm{f}}(x) n_{\mathrm{m}}(y)$. Compared with random mating, mean maternal weight increases as mating becomes more disassortative, decreases as mating becomes more assortative, and remains approximately constant when females select large males. In contrast, mean paternal weight increases 

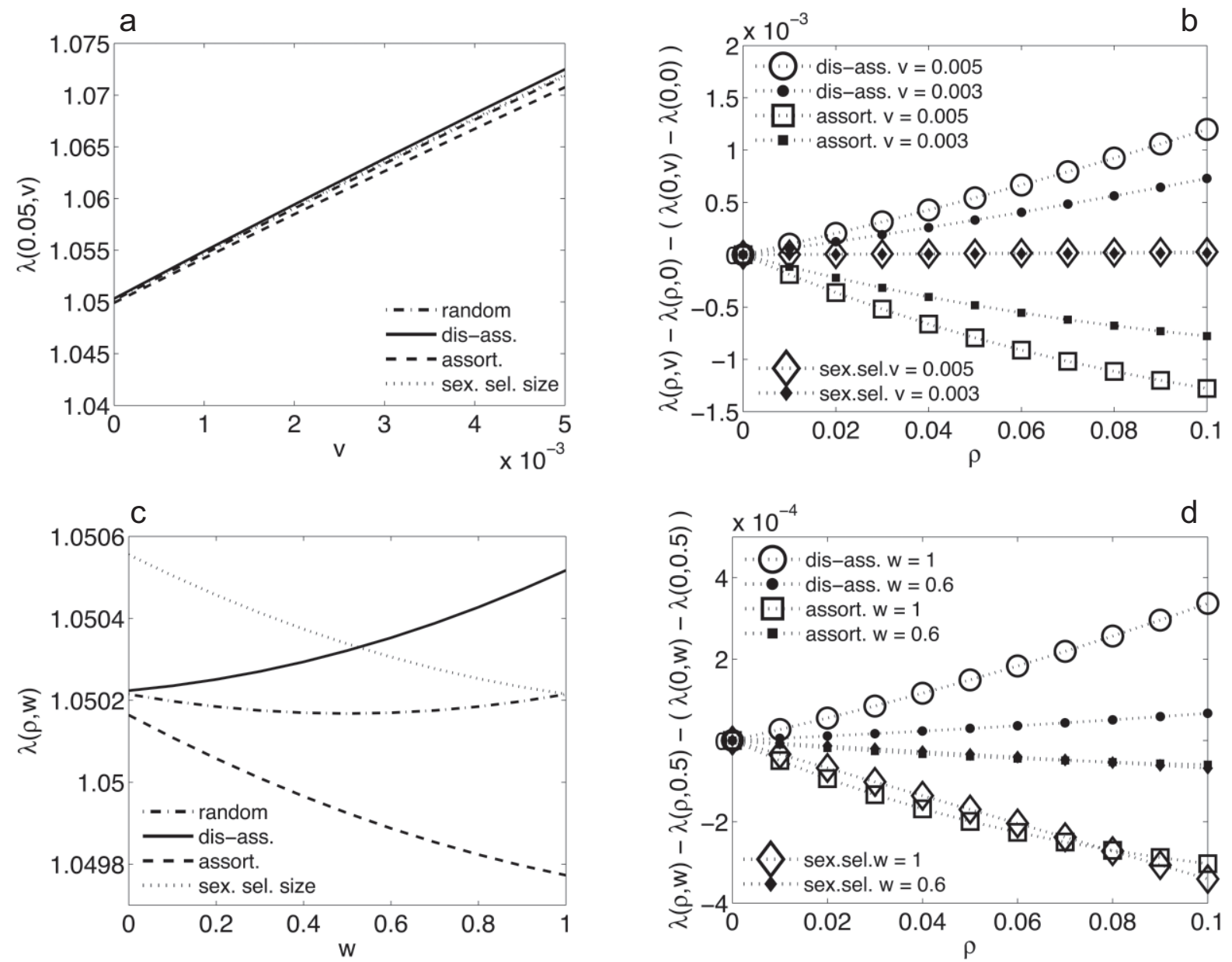

Figure 5: $a$, Growth rate $\lambda(\rho, v)$ increases with $v$ in all mating scenarios. $b$, Difference of growth rate $\lambda(\rho, v)$ when $v$ is raised from $v=$ 0 to $v=0.003$ (small markers) and from $v=0$ to $v=0.005$ (large markers) for three nonrandom mating scenarios (disassortative $=$ circle marker; assortative $=$ square; selection for size $=$ diamond). Strength, $\rho$, of nonrandom mating varies along the abscissa. The effect of nonrandom mating increases for positive $v$ for disassortative and assortative mating. The effect of selection for size is independent of v. $c$, Growth rates for all mating schemes $(\rho=0.05)$ plotted against the maternal effect $w$. If $w=0$, the father passes its trait to the offspring. If $w=1$, then the mother determines the offspring's trait. Under random mating, the growth rate as a function of $w$ is symmetric along $w=0.5$ (no maternal effects). The slope of the growth rate under selection for size and assortative mating is negative (i.e., with maternal inheritance getting stronger, $\lambda(\rho, w)$ gets smaller). The opposite is true for disassortative mating. $d$, Difference in growth rate $\lambda(\rho, w)$ when $w$ is increased from $w=0.5$ to $w=0.6$ (small markers, $60 \%$ maternal influence and $40 \%$ paternal influence) and when $w$ is increased from $w=0.5$ to $w=1$ (large markers, complete maternal control over offsprings' juvenile emergence weight). The effect of nonrandom mating is larger the more $w$ varies from 0.5 .

when females choose large males but is fairly constant for disassortative and assortative mating. Under disassortative mating, heavier females are more likely to reproduce, under assortative mating females weighing less are more likely to reproduce, and under selection for size heavier males are, not unexpectedly, more likely to reproduce. These changes in the parental weight distribution translate into differences in the distribution of offspring sizes and ultimately into differences in the population growth rate.

Interestingly, perturbing the mating behavior on its own has, in all scenarios, produced a rather small effect on $\lambda$, which is primarily caused by the quasisymmetric shape of the mating function in the case of assortative and disassortative mating (meaning the roles of females and males could be exchanged in the function without much effect; fig. $1 a, 1 b$ ). However, the effect can be as great as a $2 \%$ change in $\lambda$ when large males have a mating advantage (sexual selection for size), when males have a large influence on offspring phenotype (paternal effects, $w \approx 0$ ), and when offspring size is tightly correlated to offspring sur- 
vival. Also, nonrandom mating together with sex differences can have a big effect on the population growth rate. The link between mating behavior and sex differences in the demography may have arisen in two ways. First, the mating behavior might be responsible for sex differences; second, demographic sex differences may have arisen for reasons other than the mating behavior. There is empirical evidence for the first connection, in which the mating system influences demographic parameters, for example by reducing the survival probabilities of males (dung beetle: Kotiaho and Simmons 2003) or females (Drosophila: Fowler and Partridge 1989; bed bugs: Morrow and Arnqvist 2003). Whatever causes the link, our model applies to both cases.

The shape of our mating functions has been freely assumed and reflects general nonrandom mating scenarios, which are sufficient for our goal of monitoring the effect of nonrandom mating. For other purposes, a more accurate and species-specific mating function might be needed. This would have to be estimated from empirical counts of matings in which the traits of mating partners are observed.

If mating partners contribute differently to offspring abundance or distribution, or if they differ in demographic or developmental parameters, then nonrandom mating usually has a bigger influence on population growth compared with case in which demographic parameters are the same between the sexes. Any such disparity breaks the equality (or symmetry) of the male and female demographic functions. Given that nonrandom mating often cooccurs with demographic sex differences, the potential for nonrandom mating to influence population growth in empirical systems is large.

We studied the effect of nonrandom mating together with four symmetry breaks that are most frequently reported in mammals: reduced male survival (Toïgo and Gaillard 2003; Clutton-Brock and Isvaran 2007), faster male growth (Jarman 1983), litter sizes that are dependent on maternal weight (Albon et al. 1986), and maternal or paternal effects (Byers and Waits 2006; Maestripieri and Mateo 2009). Nonrandom mating substantially impacts the population growth rate when males and females have contrasting demographic characteristics compared with when they have the same characteristics. With the exception of growth asymmetries, all symmetry breaks that we investigated magnified the effects of nonrandom mating. This holds true in the presence of reduced male survival rates. Reduced male survival rates diminish population growth for all mating schemes, but disassortative mating and selection for size lessen the reduction of the growth rate.

Producing bigger offspring increases individual reproductive success as well as population growth rate because bigger offspring have higher survival rates. The reason why squirrels do not produce bigger offspring in general is probably because this would come at a cost to other demographic parameters. In the Columbian ground squirrel, there seems to be a trade-off between offspring size and quantity, with a trend toward relatively few but bigger juveniles per litter compared with related species. We have not included such trade-offs in our study; instead, we perturbed single parameters to pinpoint their influence. Studies that identify and incorporate trade-offs will shed additional light on population dynamics.

To conclude, we have shown that the independent effect of nonrandom mating is rather small; nevertheless, it can be important for species with low $r_{\max }$. Nonrandom mating can affect population growth rate by as much as $2 \%$ if it is connected to differences between the demographic parameters of females and males or parental contributions, although this link may have arisen due to a third factor. However, because a change in the mating pattern often implies changes in demographic parameters, this is potentially important for life-history evolution, speciation, and evolution in general. Empirical studies testing our predictions and theoretical work elucidating either the effect on offspring distribution or the effect of trade-offs will help to further clarify the link between nonrandom mating and population dynamics.

\section{Acknowledgments}

We thank H. Caswell, A. Grüning, I. Smallegange, and S. Tuljapurkar for useful discussion and J. Barthold and M. van den Hurk for constructive comments. S.S. is funded by an ERC Advanced Grant to T.C., and P.N. is funded by a Swiss National Science Foundation grant (SNF 3100AO-109816).

\section{APPENDIX A}

\section{The Columbian Ground Squirrel}

Survival and Growth. We model the dynamics of body weight. The maximal weight, $y_{\max }$, is set to be $633 \mathrm{~g}$, because it exceeds the weight of the heaviest squirrel by $10 \%$. For weight values $x \in[0,633]$, the survival function $p_{s}(x)$ is approximated by T. Coulson and P. Neuhaus (unpublished data):

$$
p_{\mathrm{s}}(x)=\frac{\mathrm{e}^{-1.85+0.007 x}}{1+\mathrm{e}^{-1.085+0.007 x}} .
$$

Figure A $1 a$ plots $p_{\mathrm{s}}(x)$. 
The probability of a squirrel growing from weight $x$ to weight $y$ is proportional to $\tilde{p}_{g}(x, y)$ (T. Coulson and $\mathrm{P}$. Neuhaus, unpublished data):

$$
\tilde{p}_{g}(x, y)=\frac{1}{\sqrt{2 \pi \sigma^{2}}} \mathrm{e}^{-(y-\mu(x))^{2} / 2 \sigma^{2}}
$$

where $\sigma^{2}=1,556$ and $\mu(x)=213+0.471 x$. To obtain a probability, we calculate $p_{\mathrm{g}}(y \mid x)=\tilde{p}_{\mathrm{g}}(x, y) / C$, such that $\int p_{\mathrm{g}}(y \mid x) \mathrm{d} y=1$ (i.e., with $C=\int \tilde{p}_{\mathrm{g}}(x, y) \mathrm{d} y$. Figure $\mathrm{A} 1 b$ plots $p_{g}(y \mid x)$.

With $p_{s}(x)$ and $p_{g}(y \mid x)$, we calculate the probability that an $x$-weight individual survives and grows to weight $y$ by $p(x, y)=p_{\mathrm{s}}(x) p_{\mathrm{g}}(y \mid x)$. The function $p(x, y)$ is applied in equations (2).
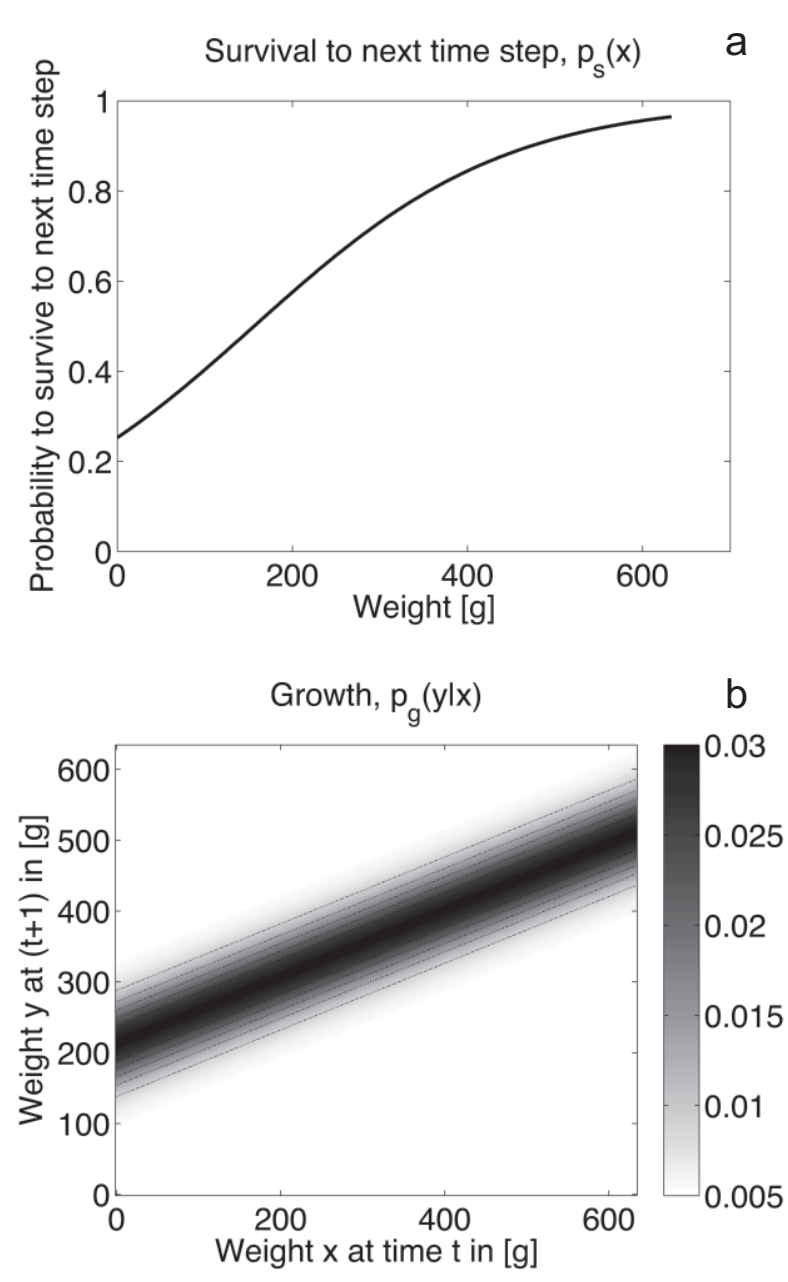

Figure A1: Composite functions of $p(x, y)=p_{s}(x) p_{g}(y \mid x)$. a, Probability of survival, $p_{\mathrm{s}}(x)$, for an $x$-weight individual given in equation (A1). b, Probability, $p_{\mathrm{g}}(y \mid x)$, of an $x$-weight individual to attain weight $y$ within one time step; see equation (A2). Plot shows color-coded probabilities (the bigger the probability, the darker the pixel) and contour lines of constant probabilities.
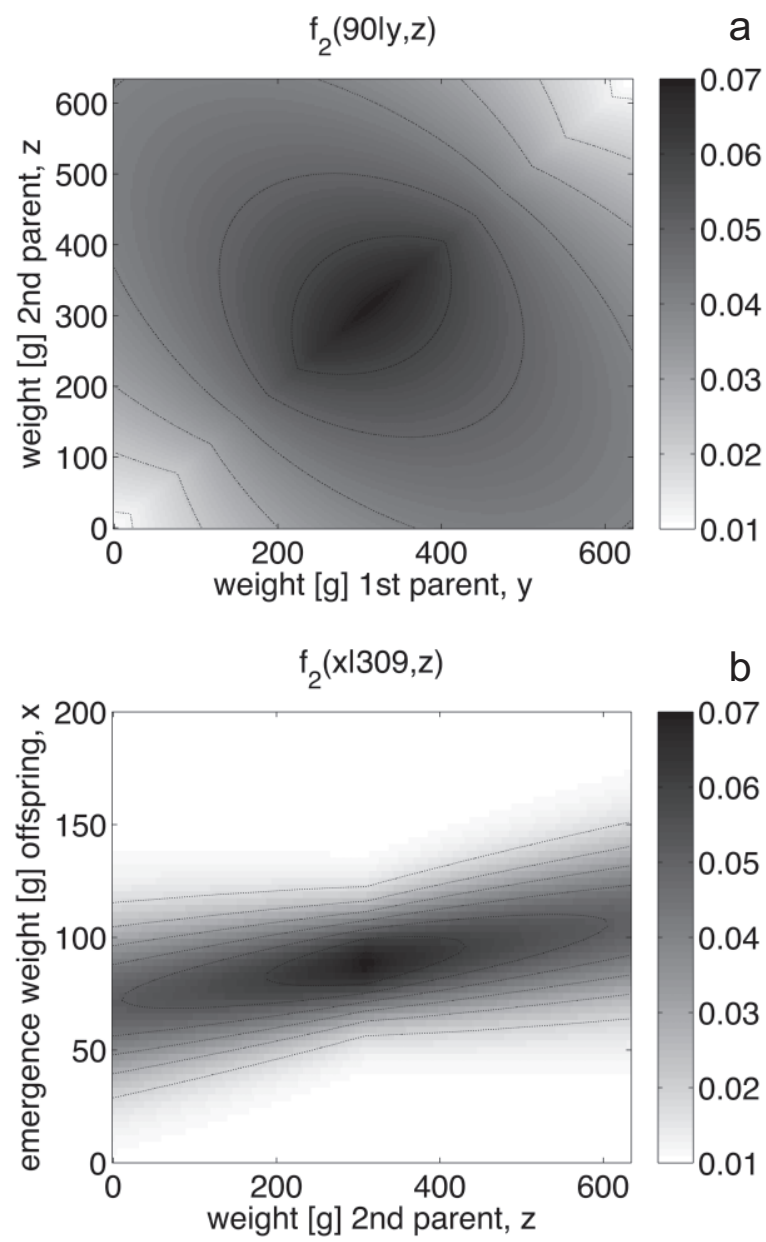

Figure A2: The inheritance function $f_{2}(x \mid y, z)$ given in equation (A5) for fixed (a) $x=90$ and (b) $y=309$. Both plots show color-coded probabilities (the bigger the probability, the darker the pixel) with contour lines of constant probabilities. $a$, Offspring weight is set to $90 \mathrm{~g}$, whereas parents' weights vary along the abscissa and ordinate. The value $f_{2}(90 \mid y, z)$ gives the probability that an offspring of parents weighing $y$ and $z$ attains a weight of $90 \mathrm{~g}$ at juvenile emergence. $b$, The first parent weighs $309 \mathrm{~g}$, whereas the weight of the second parent varies along the abscissa. The value of $f_{2}(x \mid 309, z)$ gives the probability that an offspring has juvenile emergence weight $x$ when one of its parents weighs $y=309 \mathrm{~g}$ and the other parent weighs $z$.

Reproduction. The number of offspring $R(x, y)$ that are produced by a mating pair with trait values $x$ and $y$ per breeding event is a threshold function:

$$
R(x, y)= \begin{cases}2 \times 0.87 & \text { if } x, y \geq 279 \\ 0 & \text { otherwise }\end{cases}
$$

Both parents have to weigh at least $279 \mathrm{~g}$ to mate (T. Coulson and P. Neuhaus, unpublished data).

Inheritance. We use spring juvenile emergence weight in- 
stead of birth weight because of data availability. Juveniles emerge when they are $\sim 27$ days old and their birth weight is $\sim 10 \%$ of their emergence weight. There are two reasonable possibilities to define how the offspring juvenile emergence weight $x$ is distributed, given that the weights of its parents are $y$ and $z$. First, the offspring distribution is the average of two Gaussian distributions with means determined by $y$ and $z$ and a fixed variance $\sigma_{1}^{2}=275$ :

$$
f_{1}(x \mid y, z)=\frac{1}{2 \sqrt{2 \pi \sigma_{1}^{2}}}\left(\mathrm{e}^{-\left(x-\mu_{1}(y)\right)^{2} / 2 \sigma_{1}^{2}}+\mathrm{e}^{-\left(x-\mu_{1}(z)\right)^{2} / 2 \sigma_{1}^{2}}\right),
$$

with $\mu_{1}(u)=55+0.112 u$. The inheritance function $f_{1}(x \mid y, z)$ is normalized such that $\int f_{1}(x \mid y, z) \mathrm{d} x=1$.

The second possibility is that the probability that an offspring weighs $x$ is Gaussian distributed with the mean determined by the average of its parents' weights, $(y+$ $z) / 2$, and a variance that increases with the distance $|y-z|$ :

$$
f_{2}(x \mid y, z)=\frac{1}{\sqrt{2 \pi \sigma_{2}^{2}(y, z)}} \mathrm{e}^{-\left(x-\mu_{2}(y, z)\right)^{2} / 2 \sigma_{2}^{2}(y, z)}
$$

with $\mu_{2}(y, z)=55+0.112(y+z) / 2$ and $\sigma_{2}^{2}(y, z)=$ $275+|y-z|$. Again, we normalize $f_{2}(x \mid y, z)$ such that $\int f_{2}(x \mid y, z) \mathrm{d} x=1$. Figure A2 shows $f_{2}(x \mid y, z)$ for (a) $x=90 \mathrm{~g}$ and (b) $y=309 \mathrm{~g}$.

Both inheritance functions lead to similar results; we consequently only report results when $f_{2}$ was used.

\section{APPENDIX B}

\begin{tabular}{|c|c|c|}
\hline Parameter & Description & $\begin{array}{l}\text { Value/function } \\
\text { for squirrel population }\end{array}$ \\
\hline$a, b$ & Parameters of survival probability & $-1.085, .007$ \\
\hline$c, d$ & Parameters of mean for the growth distribution & $213, .471$ \\
\hline$C_{n_{\mathrm{f}}, n_{\mathrm{m}}}$ & $\begin{array}{l}\text { Normalization constant, equivalent to number } \\
\text { of parturitions }\end{array}$ & See eq. (3) \\
\hline$e, h$ & $\begin{array}{l}\text { Parameters of mean for the offspring pheno- } \\
\text { type distribution }\end{array}$ & $55, .112$ \\
\hline$\varepsilon$ & Perturbation of survival probability & $\in[0, .5]$ \\
\hline$f(x \mid y, z), f_{1}, f_{2}, f_{2}^{\prime}$ & $\begin{array}{l}\text { Probability that newborn weighs } x \text { when its } \\
\text { parents weigh } y \text { (mother) and } z \text { (father), } f_{1} \\
\text { and } f_{2} \text { denote two options for how to set } \\
f(x \mid y, z) \text {, prime }=\text { respective probability that } \\
\text { involves maternal effects }\left(w \geq .5, \mu_{2}^{\prime} \text { replaces }\right. \\
\left.\mu_{2}\right) \text { or paternal effects }\left(w \leq .5, \mu_{2}^{\prime} \text { replaces }\right. \\
\left.\mu_{2}\right)\end{array}$ & $\mathrm{e}^{-\left(x-\mu_{1}(y)\right)^{2} / 2 \sigma_{1}^{2}}+\mathrm{e}^{-\left(x-\mu_{1}(z)\right)^{2} / 2 \sigma_{1}^{2}} / \sqrt{8 \pi \sigma_{1}^{2}}, \mathrm{e}^{-\left(x-\mu_{2}(y, z)\right)^{2} / 2 \sigma_{2}^{2}(y, z)} / \sqrt{2 \pi \sigma_{2}^{2}(y, z)}$ \\
\hline$g$ & Perturbation of growth probability & $\in[0,10]$ \\
\hline$\lambda, \lambda_{\mathrm{rm}}$ & $\begin{array}{l}\text { Asymptotic population growth rate, } \mathrm{rm}= \\
\text { random mating }\end{array}$ & \\
\hline$m(x, y), m_{\rho}^{\mathrm{a}}, m_{\rho}^{\mathrm{d}}, m_{\rho}^{\mathrm{s}}$ & $\begin{array}{l}\text { Probability of a mating between partners of } \\
\text { weights } x \text { and } y \text { is proportional to } m(x, y), \mathrm{a}= \\
\text { assortative mating, } \mathrm{d}=\text { disassortative, } \mathrm{s}= \\
\text { sexual selection for size }\end{array}$ & See eqq. (4)-(6) \\
\hline$\mu(x, y), \mu^{\prime}(x, y)$ & $\begin{array}{l}\text { Mean of growth distribution, respective per- } \\
\text { turbed mean }\end{array}$ & $c+d x, c+d x+g$ \\
\hline$\mu_{1}(x), \mu_{2}(x, y), \mu_{2}^{\prime}(x, y)$ & $\begin{array}{l}\text { Means of newborn weight distributions } f_{1} \text { and } \\
f_{2} \text {, prime }=\text { distribution allows for maternal } \\
\text { or paternal effects }\end{array}$ & $e+h x, e+h(x+y) / 2, e+h(w x+(1-w) y)$ \\
\hline$n(x, t), n_{f}(x, t), n_{m}(x, t)$ & $\begin{array}{l}\text { Number of individuals with body weight } x \text { at } \\
\text { time } t \text {, respective fractions of female and } \\
\text { male populations }\end{array}$ & \\
\hline$N_{\mathrm{t}}$ & Total population count at time $t$ & \\
\hline
\end{tabular}

Table B1: Parameter table 
Table B2: Parameter table

\begin{tabular}{|c|c|c|}
\hline Parameter & Description & $\begin{array}{l}\text { Value/function } \\
\text { for squirrel population }\end{array}$ \\
\hline$p(x, y), p_{\mathrm{f}}, p_{\mathrm{m}}$ & $\begin{array}{l}\text { Combined probability for individual of } \\
\text { weight } x \text { to survive and change weight to } \\
y \text {, respective probabilities for females and } \\
\text { males }\end{array}$ & $p(x, y)=p_{s}(x) p_{\mathrm{g}}(y \mid x)$ \\
\hline$\tilde{p}_{\mathrm{g}}(x, y), \tilde{p}^{\prime}(x), p_{\mathrm{g}}(y \mid x)$ & $\begin{array}{l}\text { Probability to change body weight from } x \text { to } \\
y \text {, tilde }=\text { not yet normalized, prime }= \\
\text { perturbed function, } p_{\mathrm{g}}(y \mid x)=\text { respective } \\
\text { normalized probability such that } \\
\int p_{\mathrm{g}}(y \mid x) \mathrm{d} y=1\end{array}$ & $1 / \sqrt{2 \pi \sigma^{2}} \times \mathrm{e}^{-(y-\mu(x))^{2} / 2 \sigma^{2}}, 1 / \sqrt{2 \pi \sigma^{2}} \times \mathrm{e}^{-(y-\mu /(x))^{2} / 2 \sigma^{2}}$ \\
\hline$p_{s}(x), p_{s}^{\prime}(x)$ & $\begin{array}{l}\text { Survival probability, respective perturbed } \\
\text { probability }\end{array}$ & $\mathrm{e}^{a+b x} / 1+\mathrm{e}^{a+b x}, \mathrm{e}^{a+b x-\epsilon} / 1+\mathrm{e}^{a+b x-\epsilon}$ \\
\hline$r$ & Parameter for offspring number & 1.74 \\
\hline$R(x, y), R^{\prime}(x, y)$ & $\begin{array}{l}\text { Number of offspring per breeding of parents } \\
\text { weighing } x \text { (mother) and } y \text { (father), re- } \\
\text { spective number that increases with } x\end{array}$ & $r, r\left(1+v\left(x-y_{\min }\right)\right)$ \\
\hline$\rho$ & $\begin{array}{l}\text { Strength of mating preference, if } p=0 \text { then } \\
\text { random mating }\end{array}$ & $\in[0, .1]$ \\
\hline$s$ & Ratio of female newborns & .5 \\
\hline$\sigma^{2}$ & Variance of growth distribution & 1,556 \\
\hline$\sigma_{1}^{2}$ & Variance of newborn weight distribution $f_{1}$ & 275.181 \\
\hline$\sigma_{2}^{2}(x, y)$ & Variance of newborn weight distribution $f_{2}$ & $\sigma_{1}^{2}+|x-y|$ \\
\hline$t$ & Discrete variable for time in years & \\
\hline$v$ & $\begin{array}{l}\text { Measures how much offspring number in- } \\
\text { creases with maternal body weight }\end{array}$ & $\in[0, .005]$ \\
\hline$w$ & $\begin{array}{l}\text { Parameter that measures maternal effects } \\
\quad(w \geq 0.5) \text { or paternal effect }(w \leq 0.5)\end{array}$ & $\in[0,1]$ \\
\hline$x, y, z$ & Continuous variable for body mass in $\mathrm{g}$ & \\
\hline$y_{\min }, y_{\max }$ & $\begin{array}{l}\text { Minimal body mass required for reproduc- } \\
\text { tion, upper limit of body mass }\end{array}$ & $279 \mathrm{~g}, 633 \mathrm{~g}$ \\
\hline
\end{tabular}

\section{Literature Cited}

Albon, S. D., B. Mitchell, B. J. Huby, and D. Brown. 1986. Fertility in female red deer (Cervus elaphus): the effects of body composition, age and reproductive status. Journal of Zoology 209:447460.

Andersson, M. 1994. Sexual selection. Princeton University Press, Princeton, NJ.

Balmer, A. 2010. Mating tactics and reproductive success in male Columbian ground squirrels (Urocitellus columbianus). $\mathrm{PhD}$ diss. Auburn University, Auburn, AL.

Bateson, P. P. G., ed. 1983. Mate choice. Cambridge University Press, Cambridge.

Brown, W. D. 1993. The cause of size-assortative mating in the leaf beetle Trirhabda canadensis (Coleoptera: Chrysomelidae). Behavioral Ecology and Sociobiology 33:151-157.

Burley, N. 1986. Sexual selection for aesthetic traits in species with biparental care. American Naturalist 127:415-445.

Burley, N., G. Krantzberg, and P. Radman. 1982. Influence of colorbanding on the conspecific preferences of zebra finches. Animal Behaviour 30:444-455.
Byers, J. A., J. D. Moodie, and N. Hall. 1994. Pronghorn females choose vigorous mates. Animal Behaviour 47:33-43.

Byers, J. A., and L. Waits. 2006. Good genes sexual selection in nature. Proceedings of the National Academy of Sciences of the USA 103: 16343-16345.

Caswell, H. 2001. Matrix population models: construction, analysis, and interpretation. Sinauer, Sunderland, MA.

Cheverud, J. M., and J. B. Wolf. 2009. The genetics and evolutionary consequences of maternal effects. Pages 11-37 in D. Maestripieri and J. M. Mateo, eds. Maternal effects in mammals. University of Chicago Press, Chicago.

Clutton-Brock, T. H., S. D. Albon, R. M. Gibson, and F. E. Guinness. 1979. The logical stag: adaptive aspects of fighting in red deer (Cervus elaphus L.). Animal Behaviour 27:211-225.

Clutton-Brock, T. H., and K. Isvaran. 2007. Sex differences in ageing in natural populations of vertebrates. Proceedings of the Royal Society B: Biological Sciences 274:3097-3104.

Crespi, B. J. 1989. Causes of assortative mating in arthropods. Animal Behaviour 38:980-1000.

Descamps, S., J. Běty, O. P. Love, and H. G. Gilchrist. 2011. Individual optimization of reproduction in a long-lived migratory bird: a test 
of the condition-dependent model of laying date and clutch size. Functional Ecology 25:671-681.

Dieckmann, U., and M. Doebeli. 1999. On the origin of species by sympatric speciation. Nature 400:354-357.

Doebeli, M., and J. C. Koella. 1994. Sex and population dynamics. Proceedings of the Royal Society B: Biological Sciences 257:17-23.

Easterling, M. R., S. P. Ellner, and P. M. Dixon. 2000. Size-specific sensitivity: applying a new structured population model. Ecology 81:694-708.

Fisher, R. A. 1999. The genetical theory of natural selection: a complete variorum edition. Oxford University Press, Oxford.

Fowler, K., and L. Partridge. 1989. A cost of mating in female fruitflies. Nature 338:760-761.

Garcia-Gonzalez, F., and L. W. Simmons. 2011. Good genes and sexual selection in dung beetles (Onthophagus taurus): genetic variance in egg-to-adult and adult viability. PLoS ONE 6:e16233.

Höglund, J., and A. Lundberg. 1987. Sexual selection in a monomorphic lek-breeding bird: correlates of male mating success in the great snipe Gallinago media. Behavioral Ecology and Sociobiology 21:211-216.

Houtman, A., and J. Falls. 1994. Negative assortative mating in the white-throated sparrow, Zonotrichia albicollis: the role of mate choice and intra-sexual competition. Animal Behaviour 48:377383.

Huber, S. K., L. F. De Lean, A. P. Hendy, E. Bermingham, and J. Podos. 2007. Reproductive isolation of sympatric morphs in a population of Darwin's finches. Proceedings of the Royal Society B: Biological Sciences 274:1709-1714.

Jarman, P. 1983. Mating system and sexual dimorphism in large terrestrial, mammalian herbivores. Biological Reviews 58:485-520.

Jennions, M. D., and M. Petrie. 1997. Variation in mate choice and mating preferences: a review of causes and consequences. Biological Reviews 72:283-327.

Kappeler, P., and C. van Schaik. 2004. Sexual selection in primates: review and selective preview. Pages 3-23 in P. Kappeler and C. van Schaik, eds. Sexual selection in primates: new and comparative perspectives. Cambridge University Press, Cambridges.

Kirkpatrick, M. 1996. Good genes and direct selection in the evolution of mating preferences. Evolution 50:2125-2140.

Kotiaho, J. S., and L. W. Simmons. 2003. Longevity cost of reproduction for males but no longevity cost of mating or courtship for females in the male-dimorphic dung beetle Onthophagus binodis. Journal of Insect Physiology 49:817-822.

Lindström, J., and H. Kokko. 1998. Sexual reproduction and population dynamics: the role of polygyny and demographic sex differences. Proceedings of the Royal Society B: Biological Sciences 265:483-488.

Lott, D. F. 1984. Intraspecific variation in the social systems of wild vertebrates. Behaviour 88:266-325.

- 1991. Intraspecific variation in the social systems of wild vertebrates. Cambridge University Press, Cambridge.

Maestripieri, D., and J. M. Mateo. 2009. Maternal effects in mammals. University of Chicago Press, Chicago.

Mays, H., and G. E. H. Hill. 2004. Choosing mates: good genes versus genes that are a good fit. Trends in Ecology and Evolution 19:554559 .
McFarland, D. 1972. Comparison of alternative marriage models. Pages 89-106 in T. Greville, ed. Population dynamics. Academic Press, London.

Møller, A. P., and R. V. Alatalo. 1999. Good-genes effects in sexual selection. Proceedings of the Royal Society B: Biological Sciences 266:85-91.

Morris, D. W. 1998. State-dependent optimization of litter size. Oikos 83:518-528.

Morrow, E. H., and G. Arnqvist. 2003. Costly traumatic insemination and a female counter-adaption in bed bugs. Proceedings of the Royal Society B: Biological Sciences 270:2377-2381.

O'Donald, P. 1960. Assortative mating in a population in which two alleles are segregating. Heredity 15:389-396.

Parker, G. A. 1983. Mate quality and mating decision. Pages 141166 in P. P. G. Bateson, ed. Mate choice. Cambridge University Press, Cambridge.

Pemberton, J., S. Albon, F. Guinness, T. H. Clutton-Brock, and G. Dover. 1992. Behavioral estimates of male mating success tested by DNA fingerprinting in a polygynous mammal. Behavioral Ecology 3:66-75.

Price, R. A., and S. G. Vandenberg. 1980. Spouse similarity in American and Swedish couples. Behavior Genetics 10:59-71.

Proulx, S. R. 2001. Female choice via indicator traits easily evolves in the face of recombination and migration. Evolution 55:24012411.

Proulx, S. R., and M. R. Servedio. 2009. Dissecting selection on female mating preferences during secondary contact. Evolution 63:20312046.

Raveh, S., D. Heg, F. S. Dobson, D. W. Coltman, J. C. Gorrell, A. Balmer, and P. Neuhaus. 2010. Mating order and reproductive success in male Columbian ground squirrels (Urocitellus columbianus). Behavioral Ecology 21:537-547.

Sadleir, R. 1969. The ecology of reproduction in wild and domestic mammals. Methuen, London.

Schindler, S. 2010. Fitness-based mating: a systematic analysis of a new preference model. PhD diss. Universität Leipzig.

Summers, K., R. Symula, M. Clough, and T. Cronin. 1999. Visual mate choice in poison frogs. Proceedings of the Royal Society B: Biological Sciences 266:2141-2145.

Thompson, C. W., N. Hillgarth, M. Leu, and H. E. McClure. 1997. High parasite load in house finches (Carpodacus mexicanus) is correlated with reduced expression of a sexually selected trait. American Naturalist 149:270-294.

Toïgo, C., and J.-M. Gaillard. 2003. Causes of sex-biased adult survival in ungulates: sexual size dimorphism, mating tactic or environment harshness? Oikos 101:376-384.

Usher, M. 1972. Developments in the Leslie matrix model. Pages 2960 in J. N. R. Jeffers, ed. Mathematical models in ecology. Blackwell Scientific, London.

Van Doorn, G. S., P. Edelaar, and F. J. Weissing. 2009. On the origin of species by natural and sexual selection. Science 326:1704-1707.

Wedekind, C., T. Seebeck, F. Bettens, and A. J. Paepke. 1995. MHCdependent mate preferences in humans. Proceedings of the Royal Society B: Biological Sciences 260:245-249.

Associate Editor: Uta Berger Editor: Troy Day 